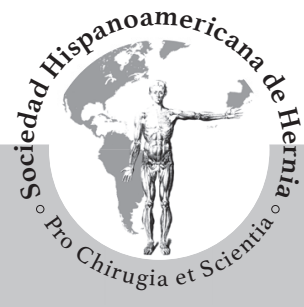

\title{
Técnica de doble cono como alternativa a la técnica de Lichtenstein en hernias inguinales tipo VI o tipo III con compromiso de la pared posterior
}

\author{
Double patch technique as an allernative to \\ Lichtenstein technique in type VI or III groin hernia \\ with posterior walll commitment
}

\section{Germán Viscido ${ }^{1}$, Facundo Mandojana ${ }^{1}$, María Cecilia Bocco Anastasía ${ }^{1}$, Matías Parodi ${ }^{1}$, Rafael Palencia', Héctor Picón Molina', Alejandro Doniquían ${ }^{2}$}

Médico del Servicio de Cirugía General. ²Jefe del Servicio de Cirugía General. Clínica Universitaria Reina Fabiola. Córdoba Capital (Argentina)

Recibido: 19-06-2017

Aceptado: 02-11-2017

\section{Palabras clave:}

Rutkow-Robbins. Técnica de

Lichtenstein. Hernias inguinales.

\section{Resumen}

Antecedentes: Rutkow y Robbins lograron aplicar su técnica a todos los tipos de hernias inguinales con excelentes resultados. La técnica de colocar dos conos de polipropileno como alternativa para la reparación de hernias tipo VI y compararla con la técnica de Lichtenstein no ha sido reportada en la literatura.

Objetivo: Evaluar los resultados a largo plazo de la técnica de doble cono (DC) para la reparación de hernias tipo VI o tipo III con compromiso de la pared posterior y compararlos con la técnica de Lichtenstein (Lch) en igual patología.

Pacientes y métodos: Estudio prospectivo y observacional. Se evaluaron los resultados de 150 hernioplastias inguinales con técnica DC en hernias tipo VI o tipo III con compromiso de la pared posterior y se los comparó con 150 hernioplastias con técnica de Lch en igual patología. Período desde 1 enero de 2008 al 1 de enero de 2015. El control se realizó a los 14, 45 y 180 días, al año y anualmente por 5 años. Se evaluaron datos demográficos, tiempo quirúrgico y de internación, complicaciones posquirúrgicas, dolor posoperatorio, reinserción laboral y porcentaje de recidiva. Programa estadístico SPSS 2008, nivel de significación del $5 \%$.

Resultados: Todos los casos se operaron con anestesia regional. No encontramos diferencias significativas al evaluar tiempo quirúrgico (DC $50 \pm 10.5$ minutos vs. Lch $48 \pm 12.8$ minutos), tiempo de internación (DC 13 vs. Lch 11 horas), morbilidad, y retorno laboral $(5 \pm 4.5$ días vs. $6 \pm 4.1$ días). En un seguimiento promedio de 48 meses encontramos 1 recidiva $(0.6 \%)$ en cada grupo $(\mathrm{p}=\mathrm{ns})$. El seguimiento pudo realizarse de forma rigurosa durante los dos primeros años en el $80 \%$ de la serie DC y en el $78 \%$ de la serie Lch.

Conclusión: La técnica de DC para hernias inguinales tipo VI o tipo III con compromiso de la pared posterior ofrece excelentes resultados en cuanto a tiempo quirúrgico y de internación, porcentaje de complicaciones, retorno laboral y recidiva como alternativa a la técnica de Lch a 48 meses de segumiento.

\section{Abstract}

Introduction: Rutkow and Robbins managed to apply their technique to all types of groin hernia with excellent results. Placing two patches of polypropylene as an alternative of repairment in type VI and III, with posterior wall commitment, groin hernia and compare it with Lichtenstein technique, has not been published yet.

Objective: The aim of this study is to evaluate and compare long term results of type VI and III, with posterior wall commitment, double patch repairment and Lichtenstein technique.

Material and methods: Prospective and observational study. One hundred and fifty type VI and III, with posterior wall commitment, double patch technique hernioplasties were evaluated and compared to 150 Lichtenstein hernioplasties with the same pathology, between January 2008 and January 2015. Post-operative controls at 14, 45, 180 days, 1 and 5 years were performed. Demographic data, surgical and admission time, post-operative complications, pain, work reintegration and recurrence rate were evaluated.

Results: When comparing surgical time ( $50 \pm 10.5$ min Double patch vs. $48 \pm 12.8$ min Lichtenstein), admission time ( 13 vs. $11 \mathrm{~h}$ ) morbidity and work reintegration ( $5 \pm 4.5$ days vs. $6 \pm 4.1)$, no significative differences were found. One recurrence in each group was found within 48 month follow up $(\mathrm{p}=\mathrm{ns})$. Two year follow up was $80 \%$ completed in double patch group and $78 \%$ in Lichtenstein group.

Conclusion: Double patch repairment in type VI and III, with posterior wall commitment, groin hernia, offers excellent results in terms of surgical and admission time, complication rate, work reintegration and recurrence as an alternative to Lichtenstein technique with 4 year follow up.

Rutkow-Robbins. Lichtenstein technique. Groin hernia. 


\section{Introducción}

La hernioplastia inguinal es una de las cirugías más frecuentes que realiza un cirujano general. Se estima que 20 millones de hernias se reparan anualmente en todo el mundo, con una tasa anual que varía entre 10 por cada 100000 habitantes en el Reino Unido y 28 por cada 100000 habitantes en EE. UU. (1).

Históricamente se clasificaron las hernias de la ingle en indirectas, directas y femorales. En los últimos 100 años fueron reportadas numerosas clasificaciones para intentar un ordenamiento de esta patología y una de las más utilizadas en la actualidad es la de Gilbert modificada que se basa en el estado de la pared posterior, diámetro del orificio profundo y la integridad de la fascia transversalis. Dicha clasificación distingue 3 tipos de hernias indirectas y dos directas y en 1993, Rutkow y Robbins (RR) incorporaron las hernias en pantalón o mixtas y las crurales (2).

Desde la descripción original, la técnica de Lch ha mostrado excelentes resultados en cuanto a porcentaje de recidiva y complicaciones posquirúrgicas, destacando su sencillez técnica, reproducibilidad y breve tiempo operatorio, entre otras razones por las cuales es una de las técnicas más utilizadas en la actualidad e incluso considerada por algunos autores como el gold estándar para el tratamiento de las hernias de la ingle (3-9).

Por otro lado, Ira M. Rutkow y Alan W. Robbins en 1993 lograron que su técnica protésica sea aplicada a todos los tipos de hernias inguinales con una recurrencia del $0.1 \%$ en más de 1500 hernioplastias, haciendo énfasis dentro de sus beneficios técnicos una incisión pequeña, mínima disección tisular, simple aprendizaje y bajo malestar posoperatorio $(10,11)$.

En hernias mixtas estos autores colocaron dos conos (DC) protésicos uno en el anillo inguinal profundo y otro en la pared posterior, suturados entre sí, técnica con resultados pocos difundidos en la literatura (11). Otros autores consideran que la técnica de RR y sus variantes, no pueden aplicarse en todos los tipos de hernias inguinales, como por ejemplo, en hernias tipo 3, particularmente en aquellas donde un voluminoso saco indirecto desplaza totalmente la pared posterior, recomendando para este tipo de hernias la técnica de $\operatorname{Lch}(12,13)$.

El objetivo de este trabajo fue evaluar los resultados a largo plazo de la técnica de DC y compararlos con la de Lch en hernias mixtas tipo VI o tipo III con compromiso total o parcial de la pared posterior.

\section{Pacientes y métodos}

Estudio prospectivo y observacional. Se evaluaron los resultados de 150 hernioplastias con DC y se los comparó con 150 pacientes con técnica de Lch en hernias tipo VI y tipo III que por su volumen comprometían la pared posterior (clasificación de Gilbert modificada por Rutkow Robbins) (2) desde el 1 enero de 2008 al 1 de enero de 2015 en el Servicio de Cirugía General de la Clínica Universitaria Reina Fabiola, Córdoba, Argentina.

Se evaluaron datos demográficos, el tiempo quirúrgico y de internación, dolor posoperatorio según EVA, recuperación laboral en días, complicaciones posquirúrgicas y porcentaje de recidiva.

En todos los pacientes se realizó antibioticoprofilaxis con Cefalotina $2 \mathrm{~g}$ endovenosos (IV). En pacientes con alergia a la penicilina se indicó Clindamicina $600 \mathrm{mg}$ IV.
La elección entre una u otra técnica fue a criterio del cirujano principal.

Se clasificó la intensidad de la carga laboral según escala de 0 a 2, siendo 0 un paciente que no tiene esfuerzo físico como desempleado o un jubilado; 1 un paciente con trabajo físicamente no exigente como trabajo de oficina y 2 un paciente con trabajo pesado con gran actividad física como construcción, mantenimiento o similar.

El dolor posquirúrgico fue evaluado según una escala visual análoga (EVA) (14), considerando un puntaje de 1 a 3 como dolor leve, 3 a 6 como moderado y 7 a 10 severo. La analgesia posoperatoria se realizó con Ketorolac 60 mg inmediatamente después de la cirugía y $30 \mathrm{mg}$ a las 5 horas de la cirugía. Luego del alta se indicó durante 5 días Diclofenac $75 \mathrm{mg}$ cada 12 horas.

En esta serie de pacientes no se realizó cirugía ambulatoria ya que contamos en nuestra institución con dicha unidad desde el año 2016.

Al alta institucional se indicó según protocolo, no manejar personalmente automóviles por 10 días, no realizar esfuerzo físico (levantar objetos que superen los 10 kilos de peso por ejemplo) o práctica de deportes por 4 a 6 semanas. Se alentó al paciente al retorno laboral precoz basándonos en el tipo de trabajo y en el esfuerzo que realiza en el mismo.

El control se realizó a los 14, 45 y 180 días, al año y anualmente por 5 años. El mismo se realizó por consultorio externo y/o por vía telefónica.

\section{Técnica de doble cono}

Se realizó una incisión de $6 \mathrm{~cm}$ paralela a la arcada inguinal tomando como reparos la espina ilíaca anterosuperior (EAS) y la espina del pubis (EP); se continuó por el tejido celular subcutáneo (TCS) y la aponeurosis del oblicuo mayor (AOM) creando en la misma dos flaps uno medial y uno lateral. Luego se individualizó sobre un reparo de látex el cordón sobre la EP. Se disecó el mesenterio del cordón liberando esta estructura totalmente, luego se incidieron las fibras del cremáster para disecar el saco indirecto que luego es invaginado a través del orificio inguinal profundo (OIP) o seccionado en muy pocos casos de acuerdo al tamaño y a la imposibilidad de reducción completa. Para la reparación del defecto y basado en el diámetro del OIP, se manufacturó un cono de polipropileno a partir de una prótesis de dicho material de $6 \times 6 \mathrm{~cm}$ u $8 \times 8 \mathrm{~cm}$ y se lo introdujo dentro del OIP. El mismo es fijado a los bordes externos del OIP con 4-6 puntos separados de Polipropileno 3.0. Para la reparación del saco directo, el mismo se disecó y posteriormente se seccionó la fascia transversalis en su base, exponiendo el tejido preperitoneal. El cono es introducido y suturado a los bordes de la fascia transversalis sana y la cintilla iliopubiana con 6-8 puntos separados de Polipropileno 3.0 en forma circunferencial y posteriormente ambos conos son consolidados con 2 puntos del mismo material obturando ambos defectos en forma completa (fig. 1). Luego se sutura la AOM con Poliglactina 3.0 y se afronta el TCS con igual material y la piel con Mononylon 3.0. Durante la cirugía se utilizan gasas embebidas en gentamicina $80 \mathrm{mg}$.

\section{Técnica de Lichtenstein}

Se realiza el mismo abordaje. Cuando se identifica el saco indirecto, este se reintroduce en el orificio inguinal profundo o se 


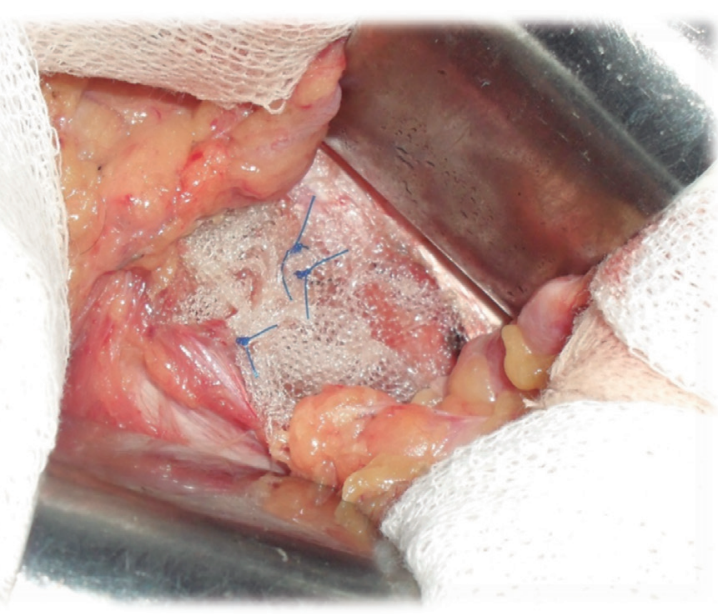

Figura 1. Técnica de doble cono en la que se visualizan ambos conos introducidos en el OIP y en la pared posterior y fijados parcialmente con Polipropileno 3.0.

secciona dependiendo el tamaño. El defecto directo es disecado y reintroducido en la pared posterior realizando un surjete con Poliglactina 3.0 borde a borde de la pared posterior (en tumores herniarios voluminosos) con el objetivo de conformar un piso para la futura prótesis y disminuir la presión en la pared posterior. A partir de una malla de polipropileno se confecciona una pieza de $6 \times 12 \mathrm{~cm}$, el extremo inferior se coloca de tal manera que sobrepase $2 \mathrm{~cm}$ la EP. Se comienza con una sutura continua de Poliglactina 3.0 comenzando en EP, tomando el borde inferior de la malla y la arcada inguinal sobrepasando $2-3 \mathrm{~cm}$ el OIP; luego se realiza un corte lateral a la malla para obtener dos bandas, la inferior más angosta (1/3) que la superior (2/3), el cordón espermático se coloca entre ambas, la banda superior se cruza con la inferior y se fijan en esta posición con 1-2 puntos de Polipropileno 3.0. El borde superior es fijado con puntos simples de Poliglactina 3.0 a la vaina del recto y al oblicuo menor con tres o cuatro puntos separados. Síntesis de AOM, TCS y piel de igual forma que en la anterior técnica.

\section{Análisis estadístico}

Se utilizó el programa estadístico SPSS 2008; las comparaciones entre tiempo quirúrgico y tiempo de internación se realizaron mediante test de Man-Whitney. Las asociaciones entre variables cuantitativas se realizaron mediante prueba chi cuadrado de independencia. Nivel de significación del $5 \%$.

\section{Resultados}

Se analizaron los resultados de 150 hernioplastias DC y se los comparó con 150 Lch realizadas en hernias tipo VI y tipo III con compromiso de la pared posterior en 300 pacientes. El $95 \%(n=285)$ fueron hernias primarias y $5 \%(n=15)$ fueron recidivadas.

En la tabla I se muestran los datos demográficos de la serie.
Tabla I. Variables demográficas analizadas

\begin{tabular}{|c|c|}
\hline Características & \\
\hline Edad promedio años (rango) & $48(25-80)$ \\
\hline Sexo n (\%) & $\begin{array}{l}\text { Masculino } 295 \text { (98.3) } \\
\text { Femenino } 5 \text { (1.7) }\end{array}$ \\
\hline Tipo de hernia n (\%) & $\begin{array}{l}\text { Hernias primarias } 285 \text { (95) } \\
\text { Hernias recidivadas } 15 \text { (5) }\end{array}$ \\
\hline $\begin{array}{l}\text { Asociación con hernia } \\
\text { umbilical } \\
\text { n (\%) }\end{array}$ & $45(15)$ \\
\hline $\begin{array}{l}\text { Asociación con hernia } \\
\text { epigástrica n (\%) }\end{array}$ & $10(3)$ \\
\hline $\begin{array}{l}\text { IMC kg/m² promedio (rango) } \\
\text { ASA I/II n (\%) } \\
\text { Puntuación de actividad } \\
\text { física laboral }\end{array}$ & $\begin{array}{c}26 \pm 8.5(19-36) \\
280(93.3)\end{array}$ \\
\hline $\begin{array}{l}n(\%) \\
0 \\
1 \\
2\end{array}$ & $\begin{array}{c}40(13.3) \\
135(45) \\
125(41.7)\end{array}$ \\
\hline
\end{tabular}

Todos los casos se operaron con anestesia regional. En este tipo de hernias por protocolo no realizamos nunca hernioplastias bilaterales en una misma cirugía.

El tiempo quirúrgico promedio en la serie DC fue de 50 minutos \pm 10.5 (rango $45-65$ ) y en la serie Lch $48 \pm 12.8$ minutos (rango 43-61) ( $\mathrm{p}=\mathrm{ns})$.

El promedio de internación en DC fue de $13 \pm 3.3$ horas (10-15) $y$ en Lch fue de $11 \pm 3.5$ horas (10-14) ( $p=n s)$.

El dolor posquirúrgico basado en EVA fue leve en el $90 \%(n=135)$ y moderado en el $10 \%(\mathrm{n}=15)$ de la serie DC. En la serie Lch el dolor fue leve en el $88 \%(n=132)$ y moderado en el $12 \%(n=18)(p=n s)$.

El retorno laboral promedio en la serie DC fue de 5 días \pm 4.5 (rango 5-10) y en la serie Lch $6 \pm 4.1$ días (rango 5-12) ( $\mathrm{p}=\mathrm{ns}$ ).

En la serie DC la morbilidad fue de $4 \%(n=6)$, incluyendo 2 seromas, 2 hematomas, una infección de sitio quirúrgico (ISO) superficial y 1 paciente con neuralgia posquirúrgica. En la serie Lch fue de $4.6 \%(n=7)$ reportando 2 seromas, 2 hematomas, una ISO superficial y 2 pacientes con neuralgias. Todas estas complicaciones fueron manejadas exitosamente con tratamiento médico sin requerir hospitalización. El análisis detallado de las complicaciones y recidiva se puede visualizar en la tabla II.

El seguimiento pudo realizarse en forma rigurosa durante los dos primeros años en el $80 \%(n=120)$ de la serie DC y en el $78 \%$ $(n=117)$ de la serie Lch. Luego de este período los controles disminuyeron significativamente su porcentaje como en la mayoría de los reportes publicados. Entre los 3 y 5 años pudo controlarse en forma personal alrededor del $30 \%$ promedio de ambas series y telefónicamente al $60 \%$. Durante este plazo se presentaron 2 recidivas $(0.6 \%$ global $)$, una en cada grupo. Un paciente de la serie Lch fue reoperado a los 4 años de la cirugía original presentando una pequeña recidiva indirecta. El otro caso no se operó por decisión del paciente ya que la recidiva era mínima y asintomática. 


\begin{tabular}{|c|c|c|}
\hline Complicación & DC & Lichtenstein \\
\hline Seroma n (\%) & $2(0.6)$ & $2(0.6)$ \\
\hline Hematoma n (\%) & $2(0.6)$ & $2(0.6)$ \\
\hline Neuralgia n (\%) & $1(0.3)$ & $2(0.6)$ \\
\hline ISO n (\%) & $1(0.3)$ & $1(0.3)$ \\
\hline Recidiva n (\%) & $1(0.3)$ & $1(0.3)$ \\
\hline $\begin{array}{l}\text { Morbilidad global } \\
\text { n (\%) }\end{array}$ & $13(4.3)$ & \\
\hline $\begin{array}{l}\text { Morbilidad por } \\
\text { técnica n (\%) }\end{array}$ & $6(4)$ & $7(4.6)$ \\
\hline
\end{tabular}

\section{Discusión}

La técnica de Lch es probablemente la más utilizada en la reparación de la hernia inguinal en nuestro medio y considerada por muchos como el gold estándar para su tratamiento (3-6,15). Recientemente la técnica laparoscópica ha mostrado muy buenos resultados en cuanto a dolor posoperatorio, recuperación, reinserción laboral y menor porcentaje de infecciones de herida operatoria demostrando ventajas en la cirugía de hernias bilaterales, recidivadas y en pacientes que requieren una pronta recuperación posoperatoria, aunque la clara desventaja del elevado costo hace que aún sea una práctica poco frecuente en nuestro medio (15-20).

Cuando se analiza una técnica quirúrgica para evaluar su efectividad en general se comparan sus resultados con una técnica estándar o con la que mejores resultados haya demostrado a través del tiempo. En la patología herniaria existen numerosas publicaciones con variadas técnicas protésicas que lograron comparar e incluso igualar sus resultados a la técnica de Lch, aun así, en la actualidad no existe una técnica que tenga claramente mejores resultados a la técnica de Lch. Dalenback et al. en su trabajo prospectivo y randomizado, comparando las 3 técnicas protésicas más frecuentes utilizadas, Prolene Hernia System (PHS), Plug-and-pach (PP) y Lch en todos los tipos de HI, no encontró diferencias significativas en cuanto a complicaciones precoces o tardías, dolor posquirúrgico y retorno laboral en 472 pacientes con seguimiento a 3 años (21). A idénticas conclusiones abordaron otros estudios (22).

Rutkow y Robbins (RR) pudieron aplicar su técnica PP con excelentes resultados en todos los tipos de hernia inguinal, como lo hizo Lichtenstein, siendo ambas técnicas protésicas en la actualidad, las más utilizadas en el tratamiento de la HI. Estos autores reportaron sus resultados en 3268 pacientes con PP en todas las clases de HI con menos de $1 \%$ de recidiva y un pronto retorno laboral $(10,11)$. Millikan et al., en 2038 hernioplastias directas e indirectas (excluyendo las mixtas) con esta técnica y seguimiento entre 2 y 10 años destaca, un corto tiempo quirúrgico, un bajo índice de recidiva $(0.15 \%)$ y dolor crónico en un $0.4 \%$ (23). Otros autores han reportado muy buenos resultados con esta técnica (24-26).

Ahora bien, ¿por qué nos planteamos el objetivo de analizar los resultados de dos técnicas quirúrgicas en dos tipos puntuales de hernias como la tipo VI o III con compromiso de la pared posterior? Consideramos que estos 2 tipos de hernias probablemente representan dentro de la patología herniaria un desafío técnico, muchas veces por su volumen o por la complejidad para su resolución. Incluso hay autores que desaconsejan utilizar otras alternativas a la técnica de Lch en hernias tipo III con compromiso de la pared posterior $(12,13)$. Aunque nuestra filosofía quirúrgica y resultados considera a la técnica de Lch como el estándar para la reparación herniaria, los resultados preliminares que obtuvimos con la utilización de conos en las hernioplastias inguinales son muy buenos, incluso transpolando estos a hernioplastias umbilicales, lo que nos alentó a aplicar esta técnica en este grupo de pacientes (27). Creemos que la forma, practicidad y lo polifacético del cono se adapta en general, a la mayoría de los anillos herniarios incluso en aquellos de gran tamaño con OIP o pared posterior severamente afectados.

No encontramos publicaciones similares utilizando la técnica del doble cono salvo algunas menciones aisladas sin resultados reportados (11). Cuando comparamos ambas técnicas no encontramos diferencias significativas entre ambas en ninguna variable. El mayor tiempo quirúrgico de la técnica DC lo atribuimos al mayor número de puntos de fijación de esta técnica. Tanto el promedio de horas de internación, el dolor posquirúrgico y el retorno laboral fueron prácticamente igual en ambas series.

Cuando analizamos las complicaciones posquirúrgicas tampoco hallamos diferencias significativas, con un porcentaje muy similar en ambos grupos ( $4 \%$ vs. $4.6 \%$ ). Todas las complicaciones fueron de baja complejidad y manejadas exitosamente con tratamiento médico. El porcentaje de complicaciones reportado con la técnica de Lch en grandes series es bajo, entre 2-6\% $(6-8,28)$. No encontramos en la literatura revisada el índice de morbilidad con la técnica de DC, pero valiéndonos del porcentaje de una técnica ampliamente difundida como la de Lch creemos que son viables nuestros resultados con la técnica de DC.

En la técnica de Lch la disección inguinal probablemente es mayor que la técnica del DC. Fue sugerido que esta disección se asoció con mayor dolor posquirúrgico y dolor crónico que otras técnicas aunque este tópico es discutido ya que la bibliografía muestra resultados dispares $(8,27,28)$. En nuestra experiencia el dolor posquirúrgico y el porcentaje de inguinodinia crónica o neuralgia posquirúrgica fue similar en ambas técnicas. Creemos que una técnica estandarizada evitando amplias disecciones innecesarias, con un manejo delicado de los tejidos, la identificación de las estructuras nerviosas de la región inguinal y la utilización de volúmenes reducidos de prótesis ayudan al menor porcentaje de neuralgias posquirúrgicas. Se podría atribuir que el uso de dos prótesis en el caso del DC podría generar mayor sensibilidad o inflamación posquirúrgica. Esta variable no fue analizada en detalle pero en general tenemos la impresión de que no es así aunque en HI voluminosas claramente se utiliza más volumen de prótesis en el DC que en Lch.

Se han reportado complicaciones propias luego de la instalación de conos entre las que se destacan la migración fuera del anillo inguinal, al escroto, vejiga y al intestino delgado o colon (29-33). Esta eventualidad, de baja frecuencia, es probablemente la que generó mayor rechazo a la utilización de los conos. Puede deberse a factores mecánicos por inadecuada fijación o fuerzas de desplazamientos externas o migraciones secundarias debidas a movimientos de la malla a través de los planos anatómicos (34). 
En nuestra experiencia no hemos observado esta complicación durante el seguimiento de estos pacientes. De todas formas, consideramos que una correcta fijación del cono con material de sutura no reabsorbible y no dejar la malla expuesta a vísceras o la vejiga disminuye la frecuencia de esta complicación.

\section{Conclusión}

La técnica de DC como alternativa a la técnica de Lch para hernias inguinales mixtas nos ofrece excelentes resultados en cuanto a tiempo quirúrgico, dolor posoperatorio, alta hospitalaria, porcentaje de complicaciones, retorno laboral y con un porcentaje bajo de recidivas en esta serie de pacientes.

\section{Bibliografía}

1. Brandi C. Tratamiento de los defectos de la pared abdominal. Rev Argent Cirug. 2009;97:2-152.

2. Zollinger R. Classification systems for groin hernias. Surg Clin North Am. 2003;83:1053-63.

3. Lichtenstein IL, Shulman AG, Amid PK, Montllor MM. The tension-free hernioplasty. Am J Surg. 1989;157:188-93.

4. Amid PK. Lichtenstein tension-free hernioplasty: its inception, evolution, and principles. Hernia. 2004;8:1-7.

5. Forte A, D’Urso A, Palumbo P, Lo Storto G, Gallinaro LS, Bezzi $\mathrm{M}$, et al. Inguinal hernioplasty: the gold standard of hernia repair. Hernia. 2003; 7:35-8.

6. Kurzer M, Belsham PA, Kark AE. The Lichtenstein repair for groin hernias. Surg Clin North Am. 2003; 83:1099-117.

7. Karaca AS, Ersoy OF, Ozkan N, Yerdel MA. Comparison of inguinal hernia repairs performed with Lichtenstein, Rutkow-Robbins, and gilbert double layer graft methods. Indian J Surg. 2015;77:28-33.

8. Dalenbäck J, Andersson C, Anesten B, Björck S, Eklund S, Magnusson O, et al. Prolene Hernia System, Lichtenstein mesh and plugand-patch for primary inguinal hernia repair: 3-year outcome of a prospective randomised controlled trial. Hernia. 2009;13:121-9.

9. Amid PK. The Lichtenstein repair in 2002: an overview of causes of recurrence after Lichtenstein tension-free hernioplasty. Hernia. 2003; 7:13-6.

10. Robbins AW, Rutkow IM. Mesh plug repair and groin hernia surgery. Surg Clin North Am. 1998;78:1007-23.

11. Doerhoff C. Technique: Plug and Puch. En: Jacob BP, Ramshaw B, editors. The SAGES Manual of Hernia Repair. Springer; 2013. pp. 50-70.

12. Millikan KW, Cummings B, Doolas A. The Millikan modified meshplug hernioplasty. Arch Surg. 2003;138:525-9; discussion 529-30.

13. Flaherty SA. Pain measurement tools for clinical practice and research. AANA J. 1996;64:133-40.

14. Iribarren C, Ribera A, Ruiz H. La hernioplastia inguinal en 2012. Encuesta a cirujanos de la Asociación Argentina de Cirugía. Rev Argent Cirug. 2013;105:45-51.

15. Tadaki C, Lomelin D, Simorov A, Jones R, Humphreys M, daSilva $\mathrm{M}$, et al. Perioperative outcomes and costs of laparoscopic versus open inguinal hernia repair. Hernia. 2016;20:399-404.
16. Takata MC, Duh QY. Laparoscopic inguinal hernia repair. Surg Clin North Am. 2008;88:157-78.

17. Bringman S, Ramel S, Heikkinen TJ, Englund T, Westman B, Anderberg B. Tension-free inguinal hernia repair: TEP versus mesh-plug versus Lichtenstein: a prospective randomized controlled trial. Ann Surg. 2003;237:142-7.

18. Simons MP, Aufenacker T, Bay-Nielsen M, Bouillot JL, Campanelli G, Conze J, et al. European Hernia Society guidelines on the treatment of inguinal hernia in adult patients. Hernia. 2009;13:343-403.

19. Poelman MM, van den Heuvel B, Deelder JD, Abis GS, Beudeker N, Bittner RR, et al. EAES Consensus Development Conference on endoscopic repair of groin hernias. Surg Endosc. 2013;27:3505-19.

20. Dalenbäck J, Andersson C. Prolene Hernia System, Lichtenstein mesh and plug-and-patch for primary inguinal hernia repair: 3-year outcome of a prospective randomised controlled trial. Hernia. 2009;13:121-9.

21. Millikan K, Doolas A. A long-term evaluation of the modified mesh-plug hernioplasty in over 2,000 patients. Hernia. 2008;12:257-60.

22. Frey DM, Wildisen A, Hamel CT, Zuber M, Oertli D, Metzger J. Randomized clinical trial of Lichtenstein's operation versus mesh plug for inguinal hernia repair. Br J Surg. 2007;94:36-41.

23. Droeser RA, Dell-Kuster S, Kurmann A, Rosenthal R, Zuber M, Metzger J, et al. Long-term follow-up of a randomized controlled trial of Lichtenstein's operation versus mesh plug repair for inguinal hernia. Ann Surg. 2014;259:966-72.

24. Fasih T, Mahapatra TK, Waddington RT. Early results of inguinal hernia repair by the 'mesh plug' technique - first 200 cases. Ann R Coll Surg Engl. 2000;82:396-400.

25. Millikan KW, Cummings B, Doolas A. A prospective study of the mesh-plug hernioplasty. Am Surg. 2001;67:285-9.

26. Viscido G, Pugliese A, Bocco M, Picón Molina H, Palencia R, Parodi $\mathrm{M}$, et al. Hernioplastias umbilicales. Análisis a largo plazo. Rev Hispanoam Hernia. 2016;4:13-9.

27. Magnusson J, Nygren J, Thorell A. Lichtenstein, prolene hernia system, and UltraPro Hernia System for primary inguinal hernia repair: one-year outcome of a prospective randomized controlled trial. Hernia. 2012;16:277-85.

28. Li J, Ji Z, Li Y. Comparison of mesh-plug and Lichtenstein for inguinal hernia repair: a meta-analysis of randomized controlled trials. Hernia. 2012;1:541-8.

29. LeBlanc KA. Complications associated with the plug-and-patch method of inguinal herniorrhaphy. Hernia. 2001;5:135-8.

30. Ishikawa S, Kawano T, Karashima R, Arita T, Yagi Y, Hirota M. A case of mesh plug migration into the bladder 5 years after hernia repair. Surg Case Rep. 2015;1:4.

31. Chuback JA, Singh RS, Sills C, Dick LS. Small bowel obstruction resulting from mesh plug migration after open inguinal hernia repair. Surgery. 2000;127:475-6.

32. Jeans S, Williams GL, Stephenson BM. Migration after open mesh plug inguinal hernioplasty: a review of the literature. Am Surg. 2007;73:207-9.

33. Dieter RA Jr. Mesh plug migration into scrotum: a new complication of hernia repair. Int Surg. 1999;84:57-9.

34. Agrawal A, Avill R. Mesh migration following repair of inguinal hernia: a case report and review of literature. Hernia. 2006;10:79-82. 